\title{
Following the thread: an unexpected cause of atrial fibrillation
}

\author{
Wiktoria Wojciechowska', Sławomir Surowiec ${ }^{1}$, Agnieszka Olszanecka', \\ Agnieszka Gawlewicz-Mroczka², Krzysztof Sładek², Danuta Czarnecka ${ }^{1}$ \\ 1 1st Department of Cardiology, Interventional Electrocardiology, and Hypertension, Jagiellonian University Medical College, Kraków, Poland \\ 2 Department of Pulmonology, Jagiellonian University Medical College, Kraków, Poland
}

Correspondence to:

Wiktoria Wojciechowska, MD, PhD, I Klinika Kardiologii i Elektrokardiologii Interwencyjnej oraz Nadciśnienia

Tętniczego, Uniwersytet Jagielloński, Collegium Medicum, ul. Kopernika 17 31-501 Kraków, Poland, phone: +48 1242473 00, e-mail: wiktoria.wojciechowska@gmail.com Received: October 12, 2016. Revision accepted: November 2, 2016 Published online: November 30, 2016 Conflict of interests: none declared. Pol Arch Med Wewn. 2016; 126 (11): 901-902 doi:10.20452/pamw.3701 Copyright by Medycyna Praktyczna Kraków 2016
A 33-year-old man, not treated before, had a history of one episode of atrial fibrillation (AF). A tumor behind the left atrium was revealed in a transthoracic echocardiogram (FIGURE 1A-C). The patient was admitted to the hospital, where a thoracic X-ray showed linear and nodular opacities in the superior segments of the upper lobes suggesting inactive tuberculosis. A thoracic computed tomography (CT) and magnetic resonance (MRI) were performed, and revealed a benign
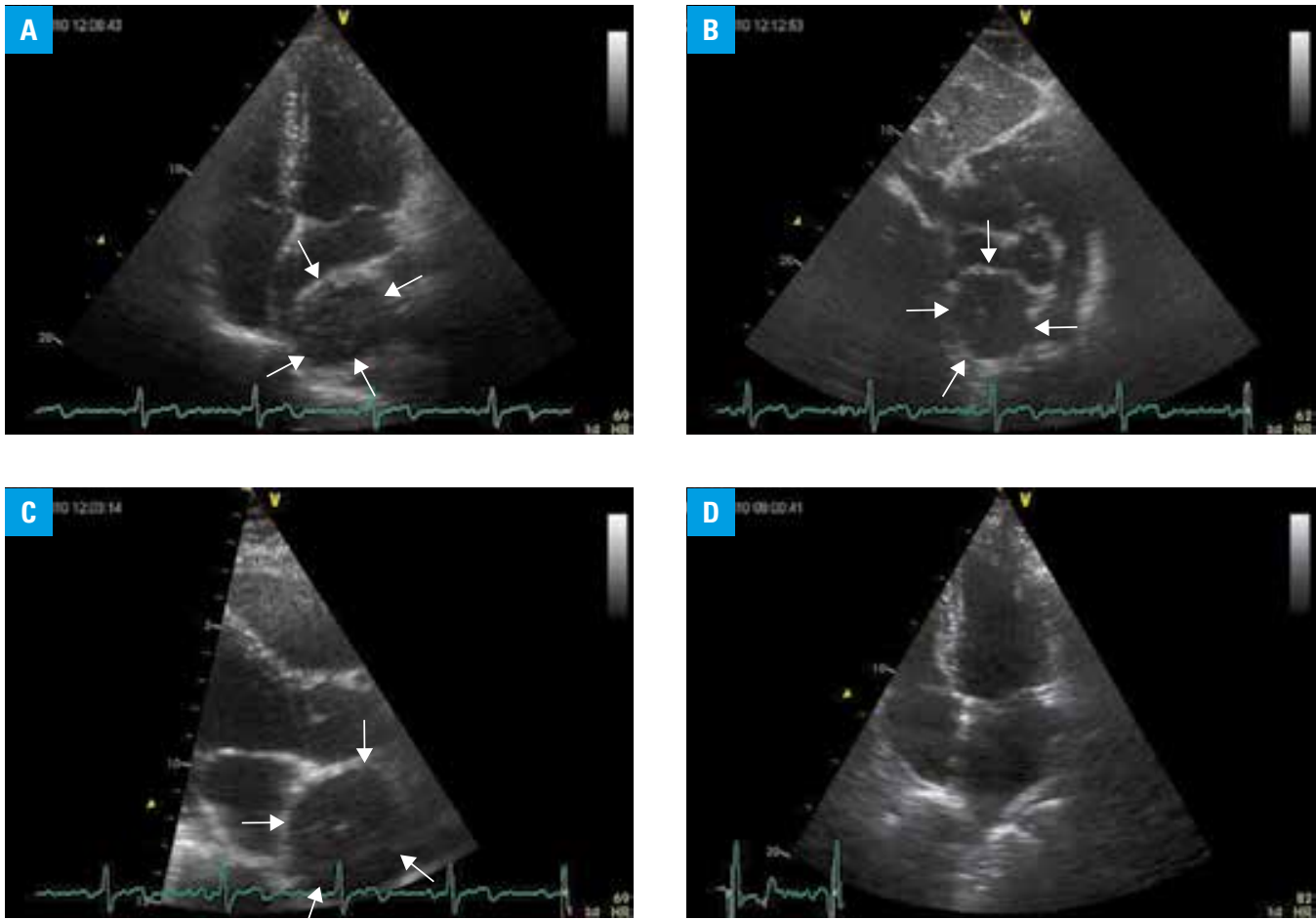

FIGURE 1 A, B, C - transthoracic echocardiograms; a 2-dimensional, 4-chamber, subcostal, and parasternal long axis view illustrating a large spherical mass adjacent to the left atrium, which caused a reduction in left atrial volume and tenting of the interatrial septum, as well as affected the shape of the right pulmonary artery; D - a follow-up echocardiogram 6 months after the operation, showing normal volume of the left atrium; E, F-a histopathological examination of the lymph node with granulation and a cyst wall, respectively. 

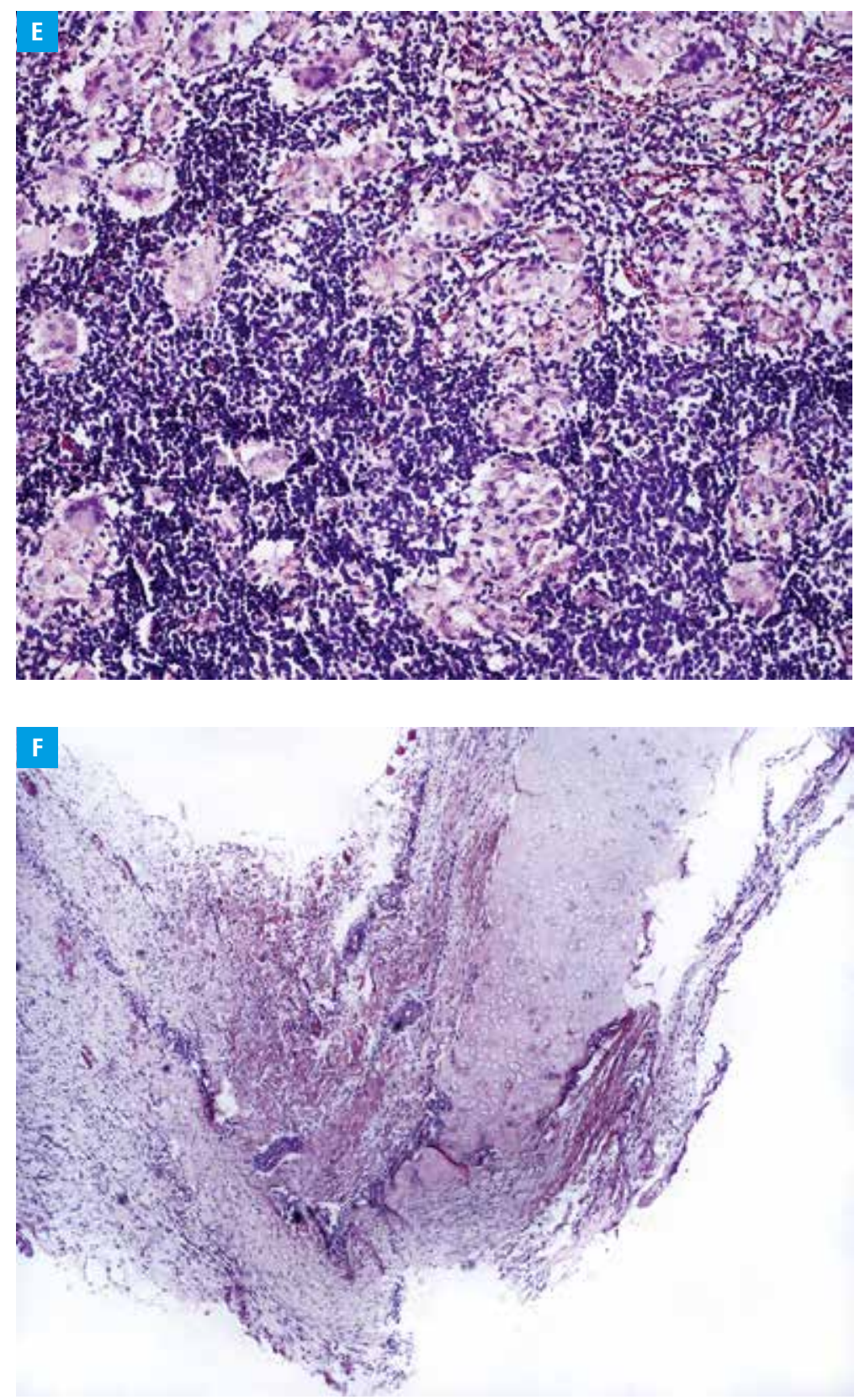

ultrasound, a bronchogenic cyst was diagnosed. A surgical removal of the cyst and of subcarinal lymph nodes was uneventful. A histological evaluation revealed that the cyst was lined by fibrous tissue, cartilage, and some histiocytic infiltration (FIGURE 1F). A histological examination of the removed lymph nodes revealed tuberculous-like granulation (without necrosis), suggesting sarcoidosis, but clinical differential diagnosis was indicated (FIGURE 1E). Tuberculosis was excluded during hospitalization in the pulmonary department and, in the second stage, sarcoidosis was diagnosed. The postoperative follow-up at 12 and then at 60 months remained uneventful, and the patient continued to have persistent sinus rhythm without symptoms of relevant rhythm disorders or disorders related to sarcoidosis (FIGURE 1D).

Cardiovascular manifestations of bronchogenic cysts have rarely been reported. ${ }^{1}$ In most cases, it was tachyarrhythmia, potentially due to compression and mechanical irritation of the heart.
A cyst could not be diagnosed easily by a thoracic radiography due to subcarinal location. ${ }^{1}$ Complete surgical resection should be recommended for all presumed mediastinal cysts, because they may become symptomatic and there have been some reports of the neoplasm occurring within these cysts. ${ }^{2}$

In sarcoidosis, cardiac involvement is unusual and can have various manifestations triggered by infiltration of the heart tissue by granulations. ${ }^{3}$ In our case, the patient suffered from asymptomatic sarcoidosis where AF was not caused by sarcoidal infiltration. According to recent guidelines for AF management, transthoracic echocardiography has only IIa class of recommendation in patients without severe symptoms, documented or suspected heart disease, or risk factors ${ }^{4}$; however, this noninvasive technique allowed identification of the real substrate of $\mathrm{AF}$ and, in the reported case, it allowed us to initiate the appropriate treatment.

Acknowledgments We would like to acknowledge the expert assistance of Dr. Lucyna Rudnicka-Sosin.

\section{REFERENCES}

1 Fujino S1, Hwang EH, Sekido N, et al. Paroxysmal atrial fibrillation due to bronchogenic cyst. Intern Med. 2010; 49: 2107-2111.

2 Okada Y, Mori $\mathrm{H}$, Maeda T, et al. Congenital mediastinal bronchogenic cyst with malignant transformation: an autopsy report. Pathol Int. 1996 46: 594-600.

3 Criado E, Sánchez M, Ramírez J, et al. Pulmonary sarcoidosis: typica and atypical manifestations at high-resolution CT with pathologic correlation. Radiographics. 2010; 30: 1567-1586.

4 Guidelines for the management of atrial fibrillation. The Task Force for the Management of Atrial Fibrillation of the European Society of Cardiology (ESC). Eur Heart J. 2010; 31: 2369-2429. 